\title{
1 A quantitative map of nuclear pore assembly reveals two distinct mechanisms
}

2

3 Shotaro Otsuka ${ }^{1,3 *}$, Jeremy O. B. Tempkin ${ }^{2}$, Antonio Z. Politi ${ }^{1,4}$, Arina Rybina ${ }^{1}$, M. Julius 4 Hossain $^{1}$, Moritz Kueblbeck ${ }^{1}$, Andrea Callegari ${ }^{1}$, Birgit Koch ${ }^{1,5}$, Andrej Sali ${ }^{2}$ and Jan Ellenberg ${ }^{1 *}$

$6 \quad{ }^{1}$ Cell Biology and Biophysics Unit, European Molecular Biology Laboratory, Meyerhofstrasse 1, 769117 Heidelberg, Germany.

$8 \quad{ }^{2}$ Department of Bioengineering and Therapeutic Sciences, Department of Pharmaceutical 9 Chemistry, Quantitative Biosciences Institute, University of California, San Francisco, San Francisco, CA 94143, USA.

11 3Present address: Max Perutz Labs, a joint venture of the University of Vienna and the Medical University of Vienna, Vienna Biocenter (VBC), Dr. Bohr Gasse 9, 1030 Vienna, Austria. ${ }^{4}$ Present address: Max Planck Institute for Biophysical Chemistry, Göttingen, Germany.

${ }^{5}$ Present address: Max Planck Institute for Medical Research, Heidelberg, Germany

*Correspondence: shotaro.otsuka@univie.ac.at; jan.ellenberg@embl.de

Summary

Understanding how the nuclear pore complex (NPC) assembles is of fundamental importance to grasp the mechanisms behind its essential function and understand its role during evolution of eukaryotes $^{1-4}$. While we know that at least two NPC assembly pathways exist, one during exit

21 from mitosis and one during nuclear growth in interphase, we currently lack a quantitative map of their molecular events. Here, we use fluorescence correlation spectroscopy (FCS) calibrated live imaging of endogenously fluorescently-tagged nucleoporins to map the changes in composition and stoichiometry of seven major modules of the human NPC during its assembly in single dividing cells. This systematic quantitative map reveals that the two assembly pathways employ 
strikingly different molecular mechanisms, inverting the order of addition of two large structural components, the central ring complex and nuclear filaments. Our dynamic stoichiometry data allows us to perform the first computational simulation that predicts the structure of postmitotic NPC assembly intermediates.

The nuclear pore complex (NPC) is the largest non-polymeric protein complex in eukaryotic cells. It spans the double membrane of the nucleus (nuclear envelope; NE) to mediate the macromolecular transport between the nucleus and the cytoplasm. To achieve this essential function, the NPC forms an octameric proteinaceous channel composed of multiples of eight of over 30 different nucleoporins (Nups) that form 6-8 protein modules, the NPC subcomplexes ${ }^{1,2}$. Therefore, more than 500 individual proteins have to come together to assemble one nuclear pore, which has the mass of tens of ribosomes. NPCs are thought to represent a key step in the evolution of endomembrane compartmentalization that allowed ancestral eukaryotes to separate their genome from the cytoplasm ${ }^{3,4}$.

In proliferating cells, there are two main pathways to assemble the NPCs. During nuclear assembly after mitosis, the NPCs form together with nuclear membranes to rapidly build new nuclei in the daughter cells (called postmitotic NPC assembly). During nuclear growth in interphase, NPCs then continue to assemble continuously for homeostasis (referred to as interphase assembly). Research over the last decade has revealed that postmitotic and interphase NPC assembly possess distinct kinetic, molecular and structural features ${ }^{5-12}$, suggesting that two fundamentally different mechanisms build the same protein complex. In postmitotic assembly, several thousand NPCs assemble within a few minutes during sealing of the initially fenestrated nuclear membranes, whereas interphase NPC assembly occurs more sporadically, requires about one hour, and involves a new discontinuity in the double membrane barrier of the NE. Studies using molecular depletions have shown that the Nup ELYS is required for postmitotic assembly 
51 but appears dispensable for interphase assembly ${ }^{5}$, whereas the membrane curvature-sensing domain of Nup1336 ${ }^{6}$ Pom121 and Sun1 $1^{7,8}$, the import of Nup153 into the nucleus ${ }^{9}$, and Torsins ${ }^{10}$ seem to be required only for interphase assembly. Recent studies correlating real time imaging with three-dimensional electron microscopy have revealed that postmitotic NPC assembly proceeds by radial dilation of small membrane openings ${ }^{11}$, while in interphase, assembly induces an asymmetric inside-out fusion of the inner and outer nuclear membranes ${ }^{12}$.

However, how several hundred proteins self-organize to form the NPC channel via these two distinct assembly pathways has remained largely enigmatic. It is technically challenging to locate the transient and rare assembly events, which has prevented investigation of the structure of assembly intermediates by either cryo-EM tomography or super-resolution microscopy. In addition, the large number of building blocks and their cooperativity often leads to complex nonlinear kinetics that can only be interpreted mechanistically using computational modelling of the structures formed during assembly. While we have some information about the dynamic addition of Nups after mitosis ${ }^{13,14}$, only sparse dynamic data is available for interphase assembly ${ }^{15,16}$. Importantly, these earlier studies could not distinguish postmitotic and interphase assemblies, whose co-occurrence in different regions of the nucleus was only discovered later; moreover, these studies provided only qualitative descriptions as they relied on ectopic expression of fluorescently-tagged Nups. Kinetic data about NPC assembly that can distinguish between the postmitotic and interphase pathways is required, including the copy numbers of Nups that assemble into forming NPCs over time. Such data would enable modeling of the assembly process and allow us to start to understand the two assembly mechanisms.

\section{Quantitative live imaging of ten nucleoporins representing seven major NPC building blocks}

To quantitatively analyze the changes in concentration of Nups at the NE during exit from mitosis and nuclear growth in G1, we genome-edited HeLa cells, homozygously tagging the endogenous 
genes for ten different Nups with mEGFP or mCherry. We chose proteins that represent the major building blocks of the fully assembled pore, including the nuclear filament protein Tpr, the nuclear Nup153, the Y-complex members Nup107 and Seh1, the central ring complex members Nup93 and Nup205, the central channel protein Nup62, the transmembrane protein Pom121 as well as the cytoplasmic filament proteins Nup214 and Nup358. Homozygous tagging was verified by careful quality control of the genome edited monoclonal cell lines ${ }^{17}$, ensuring that the tagged subunit was expressed at physiological levels, localized to the NPC and that cell viability and mitotic progression were normal (Figs. 1 and 2). The fusion proteins are likely functional, given that most Nups show strong phenotypes upon knock-out or depletion ${ }^{18}$.

To characterize the fluorescently-tagged Nups, we first performed super-resolution (STED) microscopy to determine NPC density in fully grown nuclei of the knock-in cell lines, showing that homozygous tagging had little effect on NPC density that was comparable within $15 \%$ between all cell lines with an average of 10.1 NPC per $\mu \mathrm{m}^{2}$ (Fig. 1b, c), in good agreement with our previous estimates by electron microscopy of HeLa cells ${ }^{12}$. We then used fluorescence correlation spectroscopy (FCS) calibrated confocal microscopy ${ }^{19,20}$ to determine the concentration and total number of the Nups at the NE in living cells (Extended Data Table 1, for details see Methods). Using the measured NPC density and the Nup concentration at the NE, we could calculate the average copy number of each Nup per NPC (Fig. 1d). This data showed that the investigated Nups were on average present in 16, 32, or 48 copies per pore, as expected from the eightfold symmetry of the complex and overall in good agreement with previous estimates by mass spectrometry ${ }^{21}$. This observation quantitatively validated that the homozygous tagging did not affect the incorporation of the Nups into the NPC. For Nup153 and Pom121 that exhibited lower concentrations likely due to subhomozygous tagging (Fig. 2a, Extended Data Fig. 1), we normalized their stoichiometry to the expected number of copies in the mature pore for comparison (Fig. 3a). 
NPC assembly relies on and consumes almost half of the material inherited from the mother

\section{cell within one hour after mitosis}

104 We then used our validated cell line resource to quantitatively image the Nups during both 105 postmitotic and interphase NPC assembly, from metaphase until the end of the rapid nuclear 106 growth phase in G1, two hours after anaphase onset. To this end, we performed systematic FCS107 calibrated 3D confocal time-lapse microscopy ${ }^{22}$ (Fig. 2a). Using the single molecule fluctuation 108 calibration, the 4D imaging data could be converted into maps of subcellular protein concentration

109 (Fig. 2a). Counterstaining live nuclei with SiR-DNA ${ }^{23}$, enabled computational image 110 segmentation to measure the soluble cytoplasmic pool and the NE associated pool over time ${ }^{22}$.

111 Temporal alignment to anaphase onset then allowed us to compare the dynamic association of all

112 Nups with the NE over time (Fig. 2a). Overall, the investigated Nups are present in 250,000 to $1131,200,000$ copies per human metaphase cell. After mitosis, this building material is split between

114 the daughter cells with little detectable new protein synthesis in the first hour after anaphase onset 115 (Fig. 2b). Notably, 34-53\% of the soluble pool present in the cytoplasm in metaphase was rapidly 116 re-localized to the NE during the first hour after exit from mitosis $(43,38,47,37,41,44,53$, and $11734 \%$ for Nup107, Seh1, Nups205, 93, 62, 214, Tpr and Nup358, respectively) (Fig. 2b), indicating 118 that NPC assembly initially relies almost entirely on the pool of building blocks inherited from the 119 mother cell to form the first 4,000-5,000 NPCs ${ }^{11,12}$.

121 Quantitative analysis of multimolecular kinetics of postmitotic and interphase NPC 122 assembly

123 As we and others have demonstrated previously ${ }^{12,24}$, postmitotic and interphase NPC assembly can 124 be observed in the same living cell in different regions of the NE within the first two hours after 125 mitosis. While postmitotic assembly dominates the peripheral "non-core" regions of the NE, the 
central "inner core" area is only populated with NPCs after exit from mitosis when dense spindle microtubules have been removed from the DNA surface ${ }^{25}$. Using computational segmentation and

of a fast (postmitotic) and a slow (interphase) assembly process fits the experimental data well,

131 allowing us to kinetically unmix the two assembly processes for each Nup (Extended Data Figs.

$1322 b-d$ and 3). In this way, we could for the first time perform an integrated analysis of the real time

133 kinetics of absolute concentration changes of Nups in all major NPC modules during the two

134 assembly processes at the NE (Fig. 3a). This analysis immediately revealed that the overall

135 duration of the two processes is very different, with postmitotic assembly essentially complete 15

136 min after anaphase onset, while interphase assembly only reaches a plateau after 100 min,

137 consistent with our previous estimates based on live cell imaging ${ }^{13,16}$ and correlative electron

138 microscopy ${ }^{11,12}$. Both processes reached the same final ratios between the different Nups and thus

139 presumably formed identical NPCs. However, the temporal order in which components were

140 added was distinct, including, for example, an earlier assembly of Pom 121 relative to the Y-shaped

141 complex during interphase assembly, consistent with our previous observations ${ }^{16}$.

142 To comprehensively investigate the molecular differences between the two assembly

143 processes, we relied on the constant NPC density and changes in nuclear surface area ${ }^{12}$ to convert

144 the NE concentrations of all investigated Nups into their average copy number per assembling

145 NPC over time (Fig. 3a). This result in turn enabled us to estimate changes in subunit stoichiometry

146 of the complex during its assembly in living cells. To facilitate the comparative analysis of the

147 assembly kinetics between the two pathways, in which ten components assemble with different

148 speed and order, we first reduced the dimensionality of the kinetic data. To this end, we assigned

149 a single characteristic time-point of assembly to each Nup, by sigmoidal fitting of its full kinetic

150 signature (Extended Data Fig. 4a). Plotting the copy number $v s$ the average time-point of assembly 
151 provides an overview of the major molecular differences between the two assembly pathways (Fig.

$1523 b)$.

153 While the Y-complex, Pom121, and Nup153 form a core of the first modules that assemble

154 almost simultaneously within one minute in postmitotic assembly, these components are stretched

155 out into a clear temporal order of first Nup153, second Pom121, and third the Y-complex (notably

156 with its two investigated subunits also assembling simultaneously in interphase) over more than

157 ten minutes in interphase assembly. The end of assembly on the other hand is marked for both

158 process by the addition of the large cytoplasmic filament protein Nup358. The major difference

159 was thus neither in initiation nor termination of assembly, but rather in the middle of the two

160 assembly pathways. During postmitotic assembly, the Y-complex is rapidly combined with

161 components of the central ring, building the inner core of the pore within only three minutes prior

162 to addition of either cytoplasmic or nuclear filament proteins, which follow later. In contrast,

163 during interphase assembly, the Y-complex is first combined with the nuclear filament protein Tpr

164 and the base of the cytoplasmic filament Nup214, while the central ring complex is added later.

165 This observation clearly shows that postmitotic and interphase NPC assembly not only proceed

166 with different speed but also follow a different molecular mechanism using an inverted molecular

167 order between the central ring and nuclear filaments (Fig. 3b, Extended Data Fig. 4b).

168 To obtain additional insight into the assembly mechanism, it is instructive to go beyond

169 examining the temporal order and examine our data for evidence of coupling between different

170 components of the NPC. Theoretically, for a sequential assembly mechanism where late steps

171 depend on early steps, the observed ensemble kinetics of a late binding protein must contain the

172 history of all previous events (see Methods for details). To test this prediction, we examined if one

173 or both of our assembly pathways exhibited a correlation between the average assembly time and

174 duration of the different components. We plotted the time needed for $90 \%$ of one protein to

175 accumulate in the population of assembling pores (the assembly duration) $v s$ the time when this 
176 accumulation is half maximal in the overall process (the average time-point of assembly) for all analysed Nups (Fig. 3c, Extended Data Fig. 4a). Indeed, postmitotic assembly showed a strong positive linear correlation (Fig. 3c), indicating a sequential assembly mechanism, which for example implies that Nup62 is incorporated into the NPC before Tpr can bind. In contrast,

180 interphase assembly shows poor correlation between duration and time-point of assembly (Fig.

181 3c), indicating a less strictly sequential pathway, where proteins may assemble more 182 independently of each other.

\section{Integrative modeling of the NPC assembly pathway}

185 To obtain a more comprehensive mechanistic view, we computed a spatiotemporal model of the

186 macromolecular assembly pathway, based on our dynamic multimolecular stoichiometry data in

187 combination with the available ultrastructural data about NPC assembly ${ }^{11}$ and the partial 188 pseudoatomic model of the mature $\mathrm{NPC}^{26,27}$. We modeled here only the postmitotic assembly, as

189 it showed the kinetic hallmarks of a sequential process and is known to proceed by dilating an

190 existing membrane pore with a smoothly growing proteinaceous density ${ }^{11}$. We focused on the 191 Nups contained in the structural model of the human NPC, including Nup107 and Seh1 for the Y192 complex as well as Nup93, Nup205, and Nup62 for the central ring/channel complex ${ }^{26,27}$. We 193 constrained their copy number by our stoichiometry data for the time points for which correlative

194 electron tomography data is available ${ }^{11}$, in order to use the membrane shapes and associated 195 protein densities from the tomography to constrain Nup positions. To structurally model the 196 spatiotemporal assembly process, we generalized our integrative modeling method for determining 197 static structures of macromolecular assemblies ${ }^{28,29}$. In outline (see Methods for details), we first 198 model ensembles of structures at discrete time points, then connect them between time points into 199 assembly trajectories and finally rank the alternative trajectories by fit to our data. 
We focus on the best fitting macromolecular assembly pathway that accounts for over $80 \%$ of the posterior model density (the second-scoring accounts for only 19\%). This trajectory starts by

202 formation of a single nuclear ring, composed of eight Y-complexes, concomitantly with an initial accumulation of the FG-repeat protein Nup62 and Nup205 in the center of the membrane hole

(Fig. 4). The cytoplasmic Y-complex is then added on the cytoplasmic side, before the second set of the Y-complex ring assembles on the nuclear side, again one eight-membered ring after another. In the center of the pore, Nup62 dilates from an amorphous mass into a small ring and associates membrane at early stages of assembly ${ }^{11}$. In addition, the early accumulation of Nup62 to the pore center suggests a potential role of the hydrophobic FG-repeats in the initial dilation of the small membrane hole into the larger, NPC sized channel.

\section{Discussion}

214 Our data revealed that the two assembly pathways employ strikingly different molecular 215 mechanisms. While it is at first glance surprising that the cell builds the same machine in two 216 fundamentally different ways, these distinct pathways can be rationalized by considering them in 217 the light of the rather different physiological requirements that the cell faces after mitosis and 218 during nuclear growth in interphase. Immediately after exit from mitosis, the nucleus is formed de 219 novo and the cell needs to rapidly seal its genome, exclude cytoplasm from it ${ }^{30}$ and quickly restore 220 import of essential nuclear proteins. Since the new NE originates from highly fenestrated ER 221 sheets, nuclear transport channels can be built without a formal membrane fusion requirement ${ }^{11}$.

222 The mechanism we observed for postmitotic assembly, appears well adapted to this context. The 223 rapid assembly of the nuclear Y-ring and the central ring could provide a fast way to prevent 224 complete fusion of the ER membrane holes and assemble a minimal, transport competent 
proteinaceous phase in them. Assembly of this first "transport core" NPC is complete within less than 10 min after mitosis, which precisely matches the time when transport competence is gained by postmitotic nuclei ${ }^{13,14}$.

By contrast in telo/G1 phase, the nucleus already has a sealed, double membraned NE with about 2500 postmitotically assembled and transporting pores, but needs to expand to allow the genome to take up its interphase functions. To support nuclear growth, the cell needs to maintain a constant NPC density to ensure sufficient transport capacity and maintain homeostasis to provide enough NPC building blocks for the next cell generation, which we have shown to initially rely

between the inner and outer nuclear membrane but must not disrupt nuclear integrity. The molecular pathway we observed for interphase assembly makes new predictions about its unique, inside out evaginating, mechanism. The cell first not only builds the two nuclear Y-rings but also accumulates the material for their cytoplasmic counterparts and then combines them with the nuclear filament proteins, all prior to the time of membrane fusion ${ }^{12}$. This mechanism suggests that the cytoplasmic Y-rings, including the base of the cytoplasmic filaments with Nup214, are 240 already "prebuilt" within the inner membrane evagination, where the small available volume would predict that they must be present in a very different structure than in the fully mature pore

242 after fusion. In addition, the unexpectedly early presence of Tpr suggests a key role of this large coiled-coil protein during interphase assembly, potentially in the membrane bending and/or fusion

244 process. Surprisingly, the central ring complex, which is a core structural element between the 245 nuclear and cytoplasmic Y-rings in the mature pore ${ }^{27}$ is added only later, suggesting that during 246 interphase assembly, nuclear transport control may only be added after the membrane fusion step.

247 The order of Nup assembly for interphase assembly we observed here is consistent with the 248 recently reported order of NPC assembly in budding yeast that undergoes a closed mitosis ${ }^{31}$, except 249 for the Tpr homologues Mlp1/2 which assemble late in yeast ${ }^{31}$. This difference could be due to the 
fundamental differences in cell cycle remodeling of the NE or additional functions of Mlp proteins that are believed to compensate for the absence of lamins in yeast.

Beyond the assembly mechanism the two pathways we have mapped here start to shed light

speculate that the modern NPC combines ancient membrane bending (e.g. coiled-coil filaments, such as Tpr) and membrane hole plugging (e.g. FG-repeat proteins of the central ring complex) modules, that were potentially previously used separately for extruding the cell surface or keeping transport channels open in the endomembranes around the genome. The key evolutionary innovation might lie in combining and controlling these activities, potentially by the eightmembered ring architecture of the nuclear Y-complex. In the future, comparing NPC assembly pathways in different species on the eukaryotic evolutionary tree, might help us to understand how the assembly of complex modern protein machines reflects their evolutionary origins.

\section{Main references}

2641 Hampoelz, B., Andres-Pons, A., Kastritis, P. \& Beck, M. Structure and Assembly of the $265 \quad$ Nuclear Pore Complex. Annu. Rev. Biophys. 48, 515-536 (2019).

2662 Lin, D. H. \& Hoelz, A. The Structure of the Nuclear Pore Complex (An Update). Annu. 267 Rev. Biochem. 88, 725-783 (2019).

2683 Baum, D. A. \& Baum, B. An inside-out origin for the eukaryotic cell. BMC Biol. 12, 76 $269 \quad$ (2014).

2704 Otsuka, S. \& Ellenberg, J. Mechanisms of nuclear pore complex assembly - two different 271 ways of building one molecular machine. FEBS lett. 592, 475-488 (2018).

2725 Franz, C. et al. MEL-28/ELYS is required for the recruitment of nucleoporins to chromatin and postmitotic nuclear pore complex assembly. EMBO Rep. 8, 165-172 (2007). 
2746 Doucet, C. M., Talamas, J. A. \& Hetzer, M. W. Cell cycle-dependent differences in nuclear 275 pore complex assembly in metazoa. Cell 141, 1030-1041 (2010).

2767 Talamas, J. A. \& Hetzer, M. W. POM121 and Sun1 play a role in early steps of interphase 277 NPC assembly. J. Cell Biol. 194, 27-37 (2011).

2788 Funakoshi, T., Clever, M., Watanabe, A. \& Imamoto, N. Localization of Pom121 to the 279 inner nuclear membrane is required for an early step of interphase nuclear pore complex $280 \quad$ assembly. Mol. Biol. Cell 22, 1058-1069 (2011).

2819 Vollmer, B. et al. Nup153 Recruits the Nup107-160 Complex to the Inner Nuclear 282 Membrane for Interphasic Nuclear Pore Complex Assembly. Dev. Cell 33, 717-728 (2015). 28310 Rampello, A. J. et al. Torsin ATPase deficiency leads to defects in nuclear pore biogenesis 284 and sequestration of MLF2. J. Cell Biol. 219 (2020).

28511 Otsuka, S. et al. Postmitotic nuclear pore assembly proceeds by radial dilation of small 286 membrane openings. Nat. Struct. Mol. Biol. 25, 21-28 (2018).

28712 Otsuka, S. et al. Nuclear pore assembly proceeds by an inside-out extrusion of the nuclear $288 \quad$ envelope. Elife 5 (2016).

28913 Dultz, E. et al. Systematic kinetic analysis of mitotic dis- and reassembly of the nuclear $290 \quad$ pore in living cells. J. Cell Biol. 180, 857-865 (2008).

29114 Otsuka, S., Szymborska, A. \& Ellenberg, J. Imaging the assembly, structure, and function 292 of the nuclear pore inside cells. Methods Cell Biol. 122, 219-238 (2014).

29315 D'Angelo, M. A., Anderson, D. J., Richard, E. \& Hetzer, M. W. Nuclear pores form de 294 novo from both sides of the nuclear envelope. Science 312, 440-443 (2006).

29516 Dultz, E. \& Ellenberg, J. Live imaging of single nuclear pores reveals unique assembly 296 kinetics and mechanism in interphase. J. Cell Biol. 191, 15-22 (2010).

29717 Koch, B. et al. Generation and validation of homozygous fluorescent knock-in cells using 298 CRISPR-Cas9 genome editing. Nat. Protoc. 13, 1465-1487 (2018). 
29918 Guglielmi, V., Sakuma, S. \& D'Angelo, M. A. Nuclear pore complexes in development and tissue homeostasis. Development 147 (2020).

30119 Wachsmuth, M. et al. High-throughput fluorescence correlation spectroscopy enables analysis of proteome dynamics in living cells. Nat. Biotechnol. 33, 384-389 (2015).

20 Politi, A. Z. et al. Quantitative mapping of fluorescently tagged cellular proteins using FCS-calibrated four-dimensional imaging. Nat. Protoc. 13, 1445-1464 (2018).

21 Ori, A. et al. Cell type-specific nuclear pores: a case in point for context-dependent stoichiometry of molecular machines. Mol. Syst. Biol. 9, 648 (2013).

22 Cai, Y. et al. Experimental and computational framework for a dynamic protein atlas of human cell division. Nature 561, 411-415 (2018).

23 Lukinavicius, G. et al. SiR-Hoechst is a far-red DNA stain for live-cell nanoscopy. Nat. Commun. 6, 8497 (2015). lamins. J. Cell Sci. 119, 4442-4451 (2006). envelope sealing. Nature 522, 231-235 (2015).

31526 von Appen, A. et al. In situ structural analysis of the human nuclear pore complex. Nature 526, 140-143 (2015).

31727 Kosinski, J. et al. Molecular architecture of the inner ring scaffold of the human nuclear pore complex. Science 352, 363-365 (2016).

31928 Kim, S. J. et al. Integrative structure and functional anatomy of a nuclear pore complex. Nature 555, 475-482 (2018). 1384-1403 (2019). 
bioRxiv preprint doi: https://doi.org/10.1101/2021 05.17.444137; this version posted May 18, 2021. The copyright holder for this preprint (which was not certified by peer review) is the author/funder, who has granted bioRxiv a license to display the preprint in perpetuity. It is made available under aCC-BY-NC-ND 4.0 International license.

$323 \quad 30$ Cuylen-Haering, S. et al. Chromosome clustering by Ki-67 excludes cytoplasm during nuclear assembly. Nature, 587, 285-290 (2020).

32531 Onischenko, E. et al. Maturation Kinetics of a Multiprotein Complex Revealed by Metabolic Labeling. Cell 183, 1785-1800 (2020). 
a
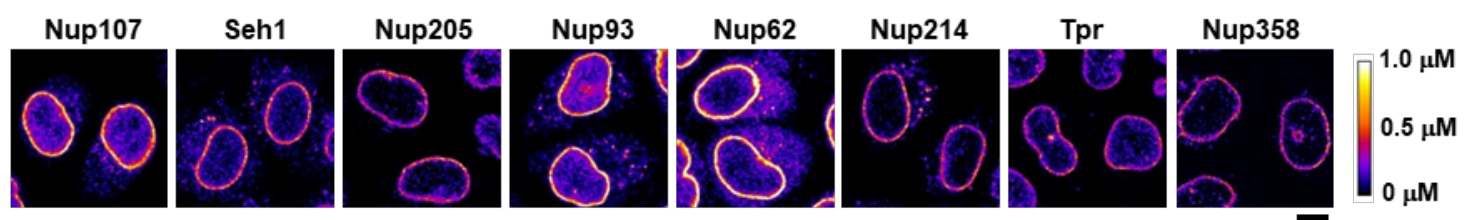

b
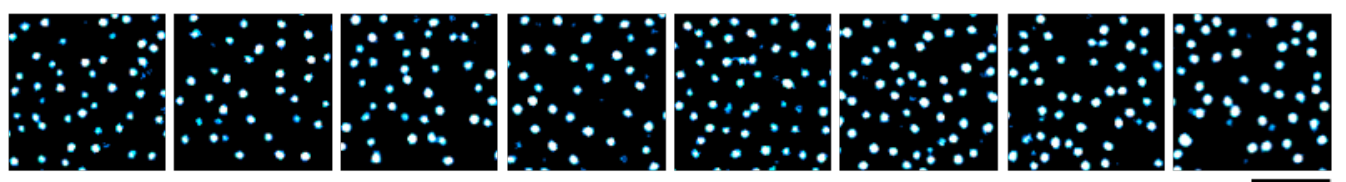

C

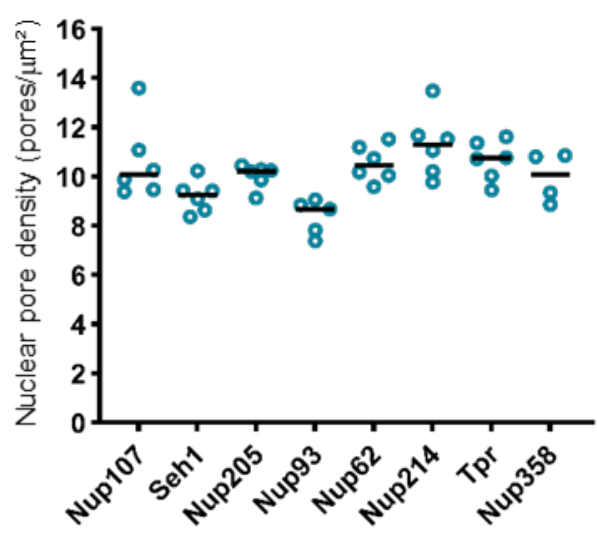

d

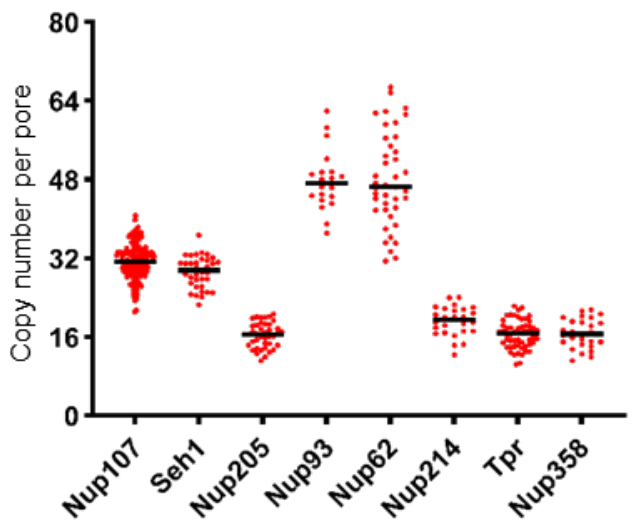

\section{Fig. 1 | Quantitative imaging of GFP-knock-in nucleoporin (Nup) cell lines.}

a, Genome-edited HeLa cells with homozygously mEGFP-tagged Nups observed by confocal microscopy. Fluorescent intensity was converted into protein concentration by FCS-calibrated imaging $^{20}$. Images were filtered with a median filter (kernel size: $0.25 \times 0.25 \mu \mathrm{m}$ ) for presentation purposes. Scale bar, $10 \mu \mathrm{m}$. b, c, Stimulated emission depletion (STED) microscopy. The genome-edited cells were stained with anti-Nup62 antibody and imaged (b), and then the density of nuclear pores was quantified (c). The plot is from 6, 6, 6, 5, 6, 6, 6, and 4 cells for Nup107, Seh1, Nup205, Nup93, Nup62, Nup214, Tpr and Nup358, respectively. Scale bar, $1 \mu \mathrm{m}$. d, Calculated copy number of Nups per nuclear pore. The plot is from 241, 37, 41, 20, 41, 26, 55, and 28 cells for Nup107, Seh1, Nup205, Nup93, Nup62, Nup214, Tpr and Nup358, respectively. The median is depicted as a line. 
bioRxiv preprint doi: https://doi.org/10.1101/2021.05.17.444137; this version posted May 18, 2021. The copyright holder for this preprint (which was not certified by peer review) is the author/funder, who has granted bioRxiv a license to display the preprint in perpetuity. It is made available under aCC-BY-NC-ND 4.0 International license.

a
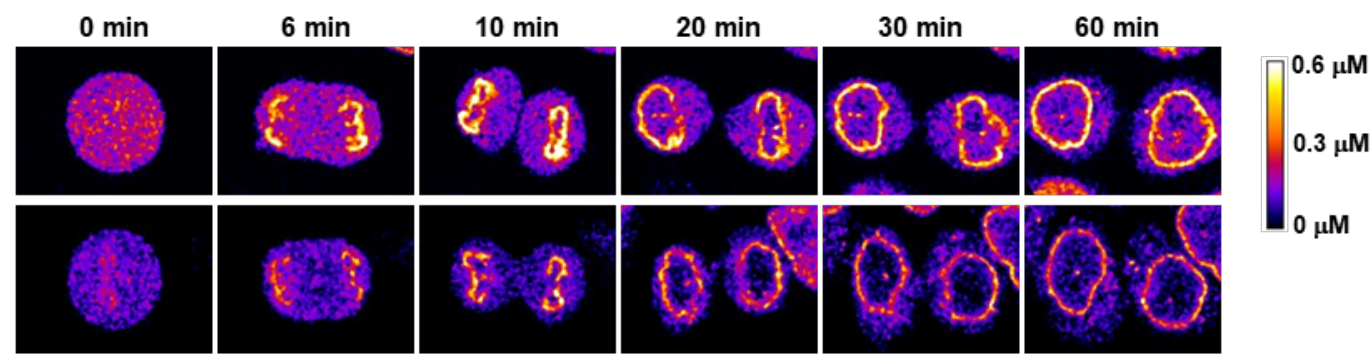

Seh1
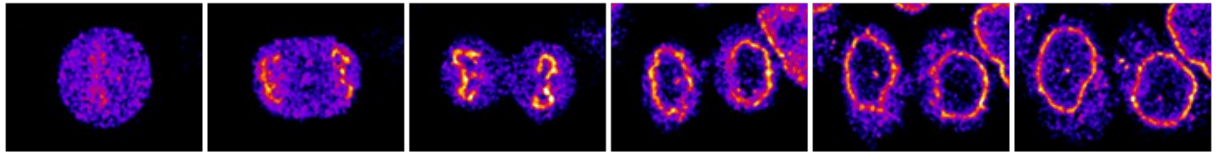

Nup205
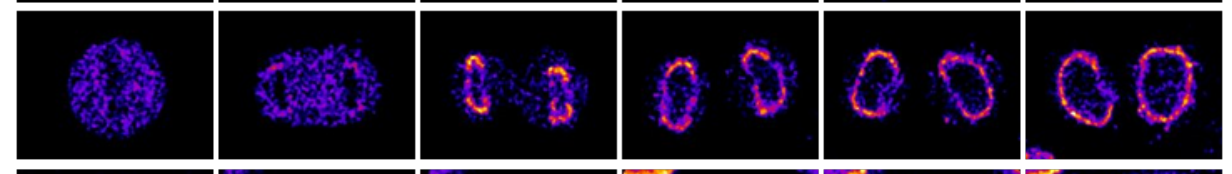

Nup93
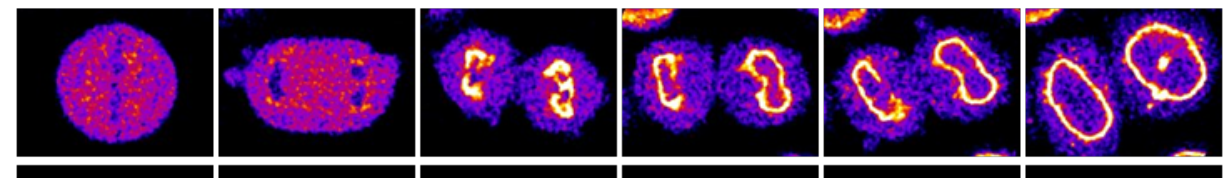

Nup62
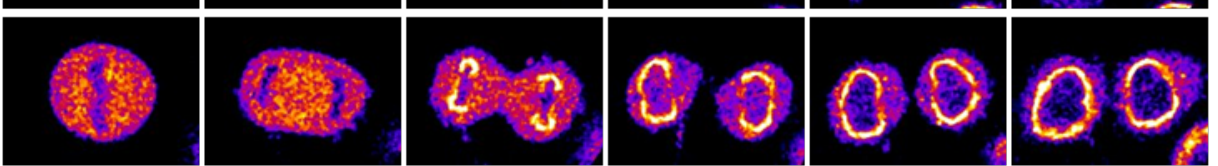

Nup214
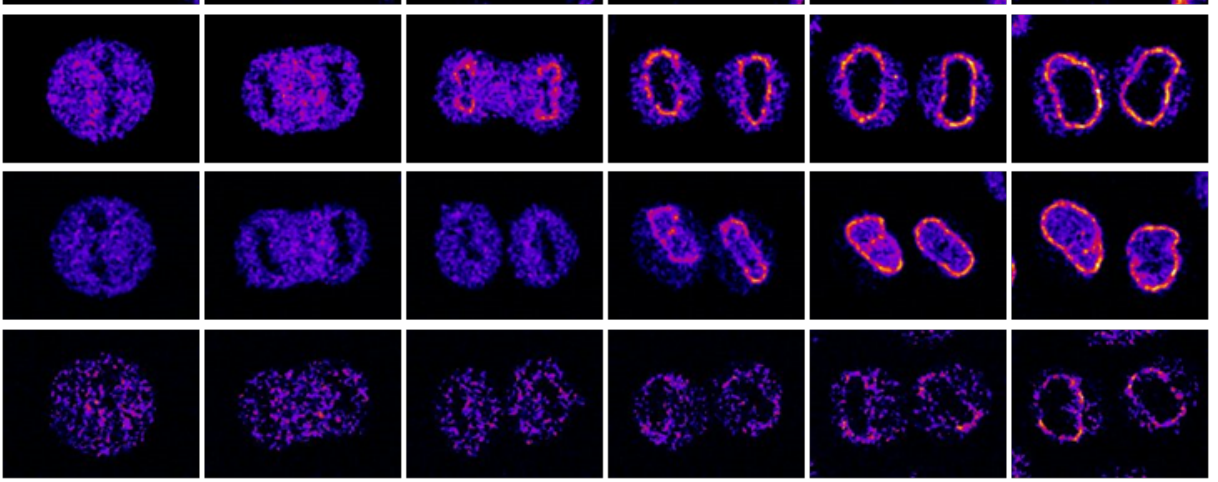

Nup153*
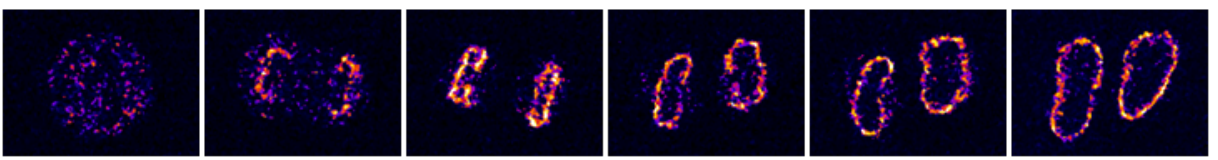

Pom121
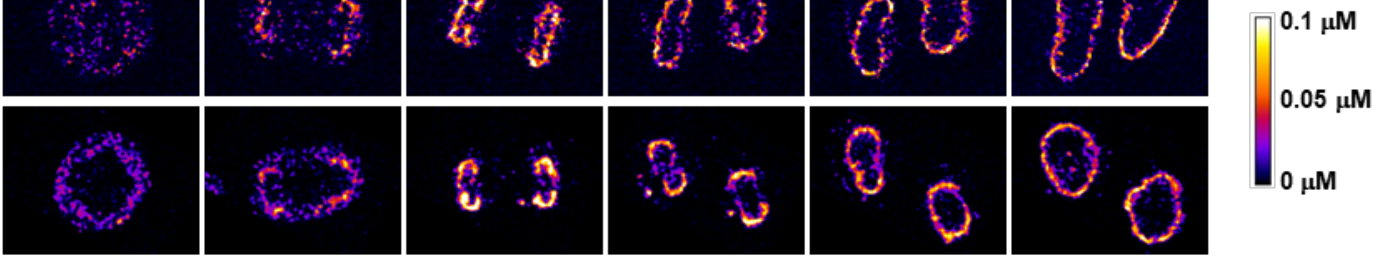

b
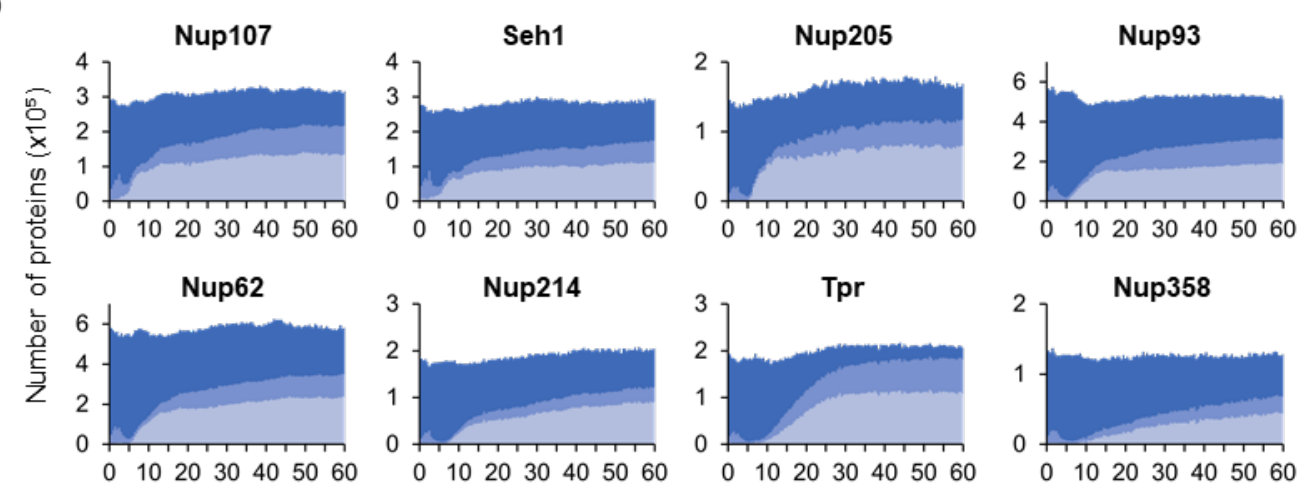

- Cytoplasm
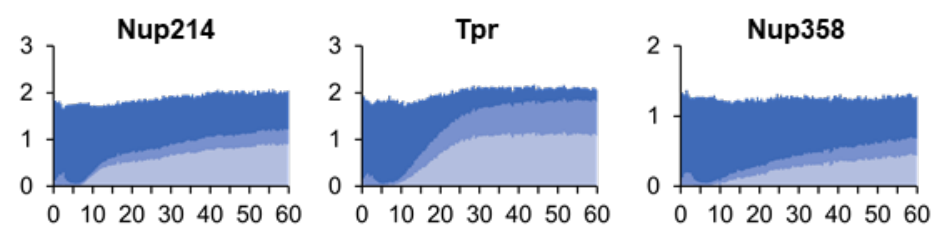

- Nucleoplasm

- Nuclear envelope

Time after AO (min) 
bioRxiv preprint doi: https://doi.org/10.1101/2021.05.17.444137; this version posted May 18, 2021. The copyright holder for this preprint (which was not certified by peer review) is the author/funder, who has granted bioRxiv a license to display the preprint in perpetuity. It is made available under aCC-BY-NC-ND 4.0 International license.

\section{Fig. 2 | Dynamic concentration maps of Nups after anaphase onset (AO).}

342 a, HeLa cells whose Nups are endogenously tagged with mEGFP or mCherry were imaged every $30 \mathrm{sec}$ by three-dimensional confocal microscopy. Single confocal sections are shown. Images were calibrated by FCS to convert fluorescence intensities into cellular protein concentration. *For Nup153 and Pom121, these Nups are not fully validated to be homozygously-tagged. Images were filtered with a median filter (kernel size: $0.25 \times 0.25 \mu \mathrm{m}$ ). Scale bar, $10 \mu \mathrm{m}$. b, Dynamic change of Nup numbers during mitotic exit. The number of Nups in cytoplasm (dark blue), nucleoplasm (medium dark blue) and nuclear envelope (light blue) are plotted against time after anaphase onset. The plot is the mean of 15, 20, 13, 14, 22, 22, 19, and 24 cells for Nup107,

350 Seh1, Nup205, Nup93, Nup62, Nup214, Tpr and Nup358, respectively. 
a
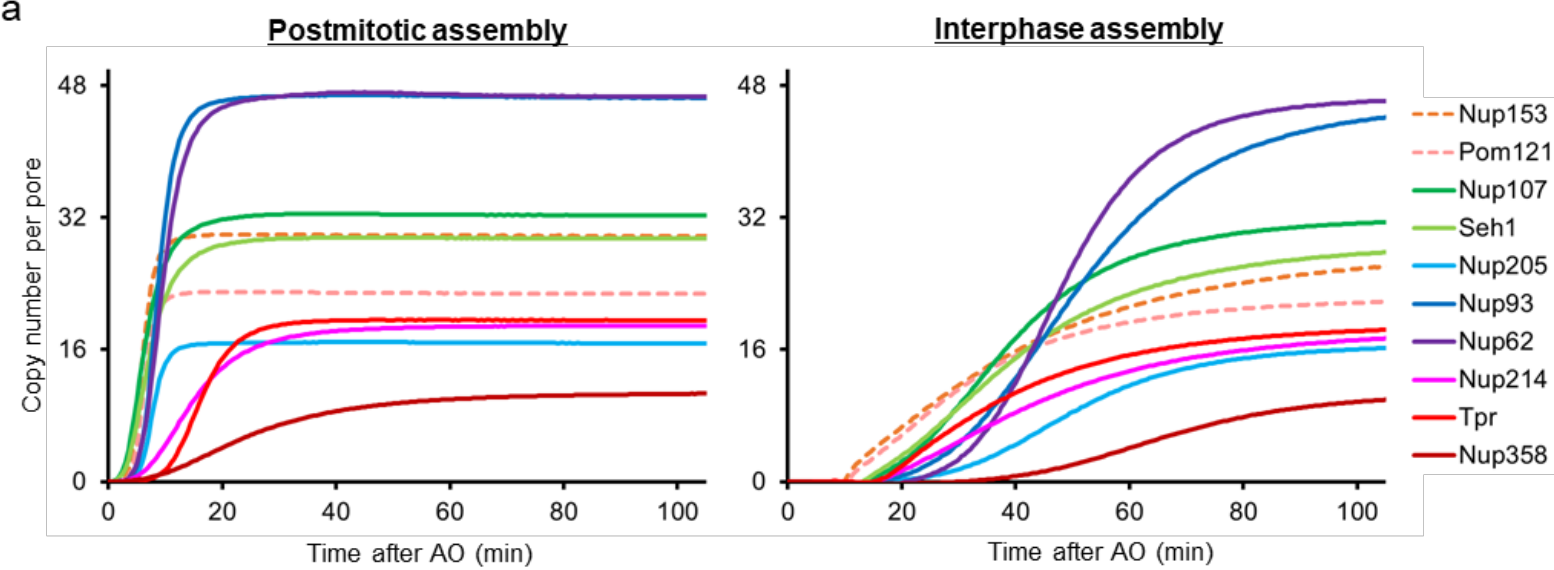

b

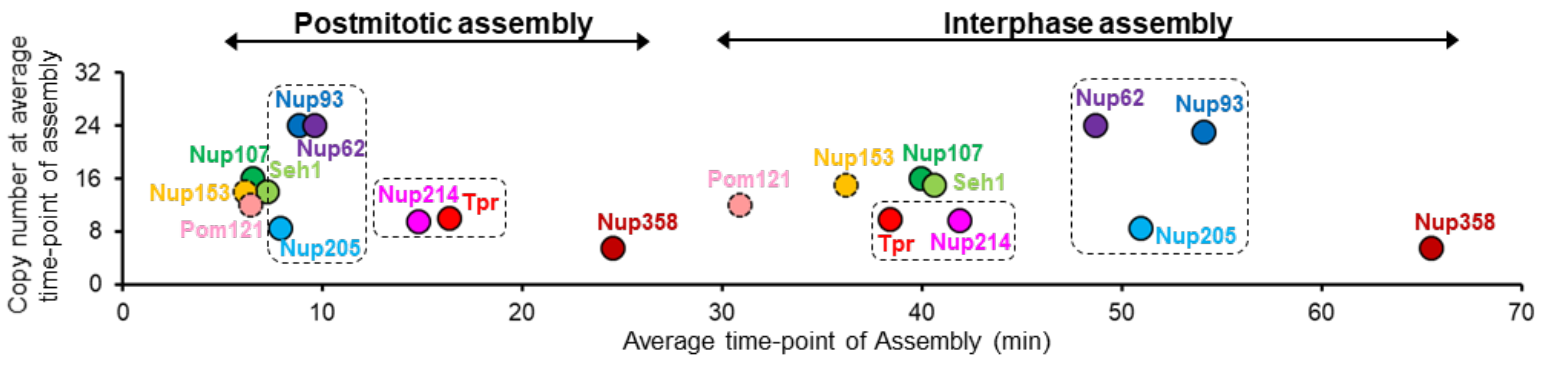

C
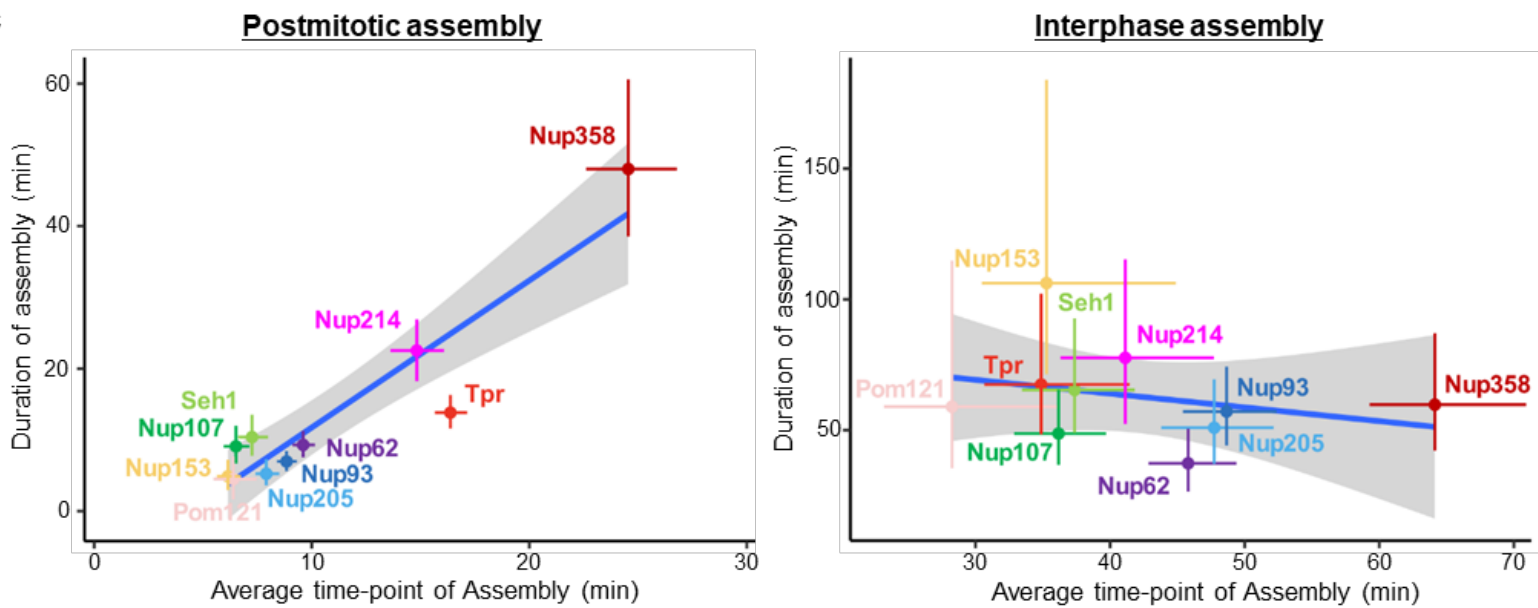

Fig. 3 | The molecular assembly order and maturation kinetics are distinct for postmitotic and interphase assembly.

a, Plots of the average copy number per nuclear pore computed from mathematical modeling for postmitotic (left) and interphase (right) assembly (see Methods and Extended Data Figs. 2 and 3 for details). For Nup153 and Pom121 (dashed lines), their absolute amount was estimated using the copy number determined from the previous study (32 for Nup153 and 16 for Pom121 $)^{21}$. b, The average copy number of individual Nups per nuclear pore are plotted along their average time-points of assembly in postmitotic and interphase assembly pathways. c, Plots of Nup assembly duration along the time-point of assembly for postmitotic and interphase assembly pathways. The crosses indicate the $95 \%$ confidence intervals. The dots are the mean values. Values are listed in Extended Data Table 2. The long straight line shows the result of a linear regression to the mean values. The gray area is the $95 \%$ confidence interval of the linear regression. 
b
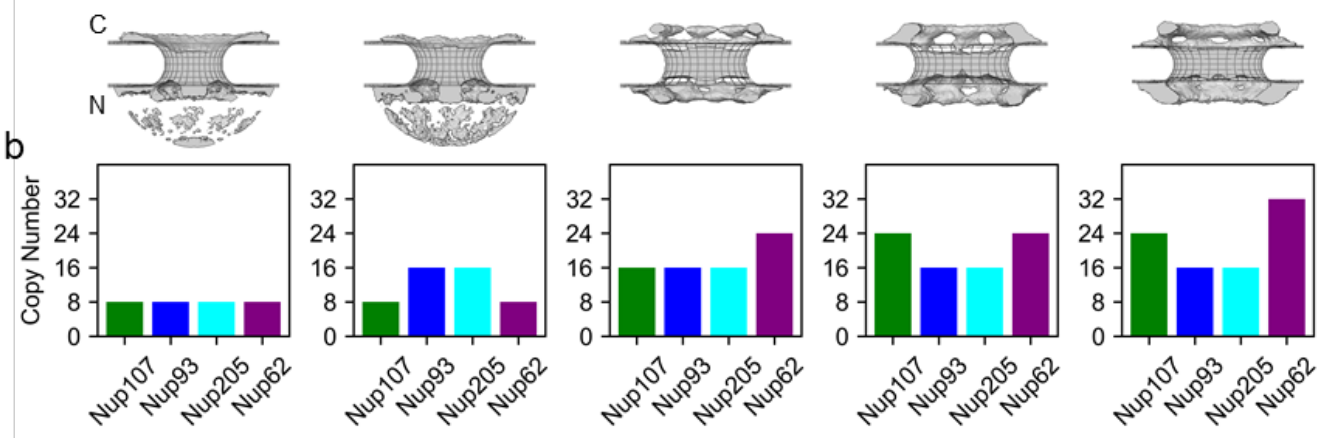

C
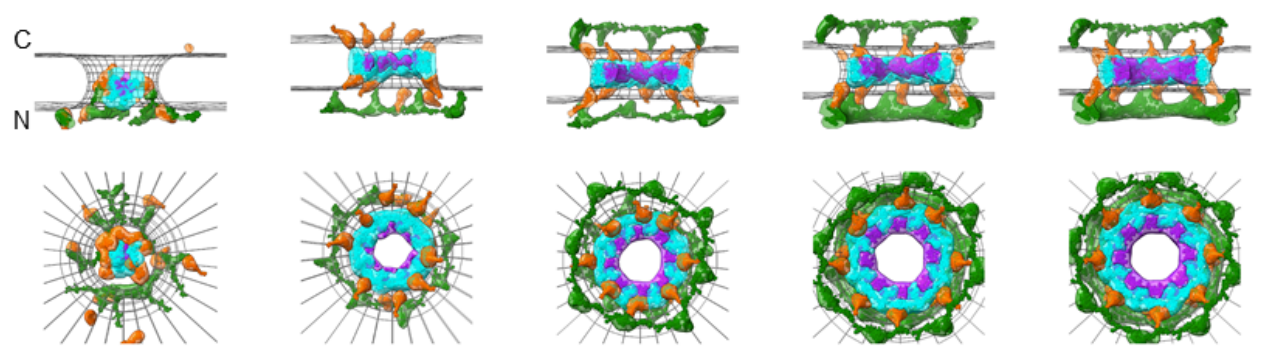
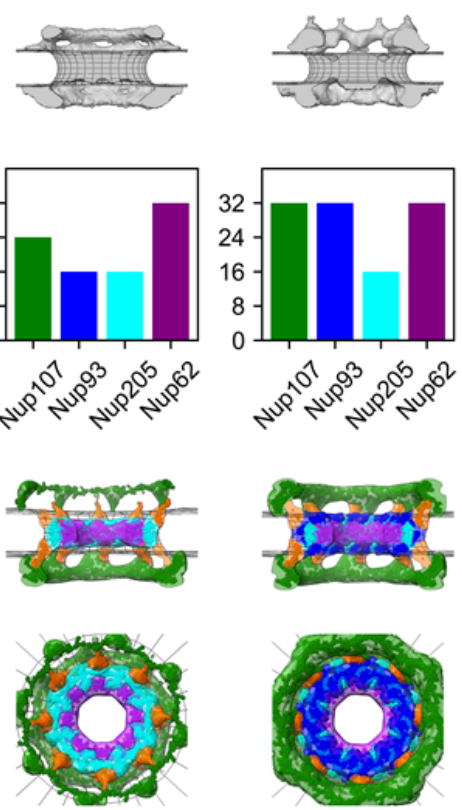

\section{Fig. 4 | Integrative model of the postmitotic NPC assembly pathway.}

a, Protein density (grey) overlaid with the NE surface (wireframe model, grey) at each time point. C, cytoplasm; N, nucleoplasm. b. Copy numbers of Nups used to construct a static NPC model at each time point. c, The best-scoring model of postmitotic assembly pathway (top and side views). The uncertainty of each Nup localization is indicated by the density of the corresponding color: Y-complex (green), Nup93-Nup188-Nup155 complex (blue), Nup205Nup93-Nup155 complex (cyan), Nup62-Nup54 complex (purple), and Nup155 (orange). 


\section{Methods}

374

375

376

377

378

379

380

381

382

383

384

385

386

387

388

389

390

391

392

393

394

395

396

397

\section{Cell culture}

Wildtype HeLa kyoto cells (RRID: CVCL_1922) were kind gift from Prof. Narumiya in Kyoto University, and the genome was sequenced previously ${ }^{32}$. Cells were grown in high glucose Dulbecco's Modified Eagle's Medium (DMEM) containing 4.5 g/l D-glucose (Sigma Aldrich, St. Louis, MO) supplemented with 10\% fetal calf serum (FCS), $2 \mathrm{mM}$ l-glutamine, $1 \mathrm{mM}$ sodium pyruvate, and $100 \mu \mathrm{g} / \mathrm{ml}$ penicillin and streptomycin at $37{ }^{\circ} \mathrm{C}$ and $5 \% \mathrm{CO}_{2}$. The mycoplasma contamination was inspected by PCR every 2 or 3 months and was always negative.

\section{Genome editing}

Monomeric enhanced GFP (mEGFP) and mCherry were inserted into the genome using zinc finger nucleases or CRISPR-Cas9 nickases ${ }^{17}$. The following six cell lines had been generated and published previously: Nup62-mEGFP ${ }^{33}$, mEGFP-Nup107 ${ }^{12}$, mEGFP-Nup205 ${ }^{11}$, mEGFP-Nup214, mEGFP-Nup358 (also called RanBP2) and Tpr-mEGFP'22. The following four cell lines were generated in this study: mEGFP-Seh1, Nup93-mEGFP, mEGFP-Nup153, and Pom121-mCherry. The gRNA sequences used for generating these cell lines are summarized in Extended Data Table 3. For the Nup93-mEGFP cell line, CRISPR-Cas9 nickases and the donor plasmid were transfected by electroporation (Neon Transfection System, Thermo Fisher Scientific, Waltham, MA) instead of a polymer-mediated transfection reagent.

\section{FCS-calibrated live-cell imaging and estimation of Nup copy numbers per NPC}

(i) Wild-type cells, (ii) wild-type cells transfected with mEGFP using Fugene6 (Promega, Madison, WI), (iii) mEGFP-Nup107 genome-edited cells, and (iv) the cells of another mEGFPNup genome-edited cell line, were seeded on each well of 8-well Lab-Tek Chambered Coverglass (Thermo Fisher Scientific). On the day of live-cell imaging, DMEM was replaced by imaging 
medium: $\mathrm{CO}_{2}$-independent medium without phenol red (Invitrogen) containing 20\% FCS, $2 \mathrm{mM}$ enclosing incubator at $37^{\circ} \mathrm{C}$ for at least 30 min before imaging.

Calibrated imaging using fluorescence correlation spectroscopy (FCS) was carried out as described in a previous report ${ }^{20}$. Briefly, the confocal volume was determined by performing FCS mCherry). To convert fluorescence intensity to the concentration, FCS was performed in the cells that transiently-express mEGFP or mCherry alone. Then a calibration curve was obtained by plotting the fluorescence intensity along the concentration. The background fluorescence signal was measured in cells without expressing fluorescent proteins and subtracted.

To measure the concentration of Nups, mEGFP-Nup genome-edited cells in interphase were 411 imaged in 3D using a confocal microscope (LSM780; Carl Zeiss, Oberkochen, Germany) and a $40 \times 1.2$ NA C-Apochromat water immersion objective (Carl Zeiss) at $37^{\circ} \mathrm{C}$ in a microscope-bodyenclosing incubator, under the following conditions: 21 optical sections, section thickness of 2.0

$414 \mu \mathrm{m}, \mathrm{z}$-stacks of every $1.0 \mu \mathrm{m}$, and the xy pixel size of $0.25 \mu \mathrm{m}$. When the NE is not perpendicular 415 to the confocal plane of the 3D stacks, the fluorescence intensity at the NE is nonisotropic in the 416 point-spread function (PSF), which results in underestimation of the signal. To avoid such 417 underestimation, a single plane was selected that contains the largest nuclear area in which the NE 418 is perpendicular to the imaging plane and thus isotropic in the PSF. The fluorescence intensity of 419 Nups was quantified on this single plane using the NE mask with the width of three pixels $(0.75$ $420 \mu \mathrm{m})$ that was generated from a SiR-DNA channel. Background fluorescence intensity was 421 measured in wild-type cells without expressing any fluorescent proteins and subtracted. The Nup 422 fluorescence intensity on the NE was converted to the concentration using the calibration curve 
generated by FCS above. The number of Nups per square micro-meter was calculated from the concentration and then divided by the NPC density per square micro-meter measured by stimulated emission depletion (STED) microscopy. This absolute quantification of Nup copy number with FCS calibration was done using 47 mEGFP-Nup107 genome-edited cells in interphase. For other mEGFP-Nups genome-edited cells, their Nup fluorescent intensities on the NE were directly compared with the ones of mEGFP-Nup107 genome-edited cells on the same 8-well Lab-Tek Chambered Coverglass, and then their concentrations were determined using the intensity ratios to the mean intensity of mEGFP-Nup107 without using a FCS calibration curve. For Pom121mCherry, the copy number was quantified independently.

\section{Measurement of nuclear pore density by stimulated emission depletion (STED) microscopy}

For STED super-resolution imaging, cells were fixed with $2.4 \%$ formaldehyde (Electron Microscopy Sciences, Hatfield, PA) in PBS for 10 min, extracted with 0.4\% Triton X-100 (Sigma Aldrich) in PBS for 5 min, and blocked with 5\% normal goat serum (Life Technologies, Carlsbad, CA) in PBS for 10 min at room temperature. Subsequently, the cells were incubated overnight at $4^{\circ} \mathrm{C}$ with a mouse anti-Nup62 (Cat. No. 610497; BD Biosciences, Franklin Lakes, NJ) antibody, and then with an Abberior ${ }^{\circledR}$ STAR RED-conjugated anti-mouse IgG (Cat. No. 2-0002-011-2, Abberior GmbH, Göttingen, Germany) for $30 \mathrm{~min}$ at room temperature. After multiple washes in PBS, cells were mounted in Vectashield (Cat. No. H-1500, Vector Laboratories Inc., Burlingame, CA). Super-resolution imaging was performed on a Leica SP8 3X STED microscope as described in a previous report ${ }^{12}$. The images were taken with a final optical pixel size of $20 \mathrm{~nm}, \mathrm{z}$-stacks of every $250 \mathrm{~nm}$, and the optical section thickness of $550 \mathrm{~nm}$. Images were filtered with a Gaussian filter (kernel size: $0.5 \times 0.5$ pixel) for presentation purposes. The shrinkage of the nucleus by formaldehyde fixation was quantified by comparing the volume of the nuclei of live cells with the ones of fixed cells. The shrinkage was $9.1 \pm 2.6 \%$ (the average and standard error, $\mathrm{N}=36$ cells). 
448 The NPC density was corrected for the nuclear shrinkage for the calculation of Nup copy number 449 per NPC in Fig. 1d.

To quantify NPC density, the raw STED data were processed in ImageJ

451 (http://rsbweb.nih.gov/ij/) with a mean filter (kernel size: $2 \times 2$ pixels) and a sliding paraboloid 452 (radius: 5 pixels) for background subtraction. Detection of central peak positions for individual 453 NPCs was carried out with the plugin TrackMate ${ }^{34}$, using DoG detector and adjusting the detection 454 threshold as the spot diameter size. The resulting 3D NPC coordinates were used to visualize and 455 determine flat and curved regions of the nucleus. Using this map, circular and ellipsoidal ROIs 456 could then be selected in the flatter parts containing central NPC positions within the Z-depth of 457 approx. $500 \mathrm{~nm}$, which corresponds to $2-3$ microscopic slices in the images. The remaining signal 458 outside the ROIs, as in curved regions or cytoplasmic structures were discarded from further analysis. NPC densities were calculated for each cell separately by dividing the number of NPCs 460 within the selected ROIs by the corresponding ROI areas. For each cell line, the values were 461 combined to calculate the mean and median NPC density values.

Quantification of Nup copy number in the cytoplasm and the nucleoplasm as well as in noncore and core regions of the $\mathrm{NE}$

465 Mitotic cells were imaged and monitored from anaphase onset for two hours in 3D by confocal 466 microscopy. The microscopy setup and the imaging conditions are described above. Time-lapse 467 imaging for mEGFP-tagged Nups was performed every $30 \mathrm{sec}$. Photobleaching was negligible and 468 thus not corrected. Time-lapse imaging for Pom121-mCherry was carried out every 60 sec, and 469 photobleaching was corrected by measuring a fluorescence signal decay in a neighboring cell in 470 the same field of view. Visualization of the chromosome surface in 3D was done in the Amira 471 software package ${ }^{35}$. 
To measure the Nup accumulation on the NE, single planes were selected that contain the largest nuclear area at individual time points to avoid underestimation of the signal as mentioned earlier. The Nup intensity was quantified on the NE mask with the width of $0.75 \mu \mathrm{m}$ that was generated from a SiR-DNA signal at each time point. Except for Nup107 and Seh1, the Nup signal in the cytoplasm and nucleoplasm was measured and used as background. For Nup107 and Seh1, only the cytoplasmic signal was used as background because of their localization at kinetochores. multiplied with nuclear surface area to calculate the total number of the Nups on the NE. For

Nup153 and Pom121, we did not convert the fluorescence intensity to the concentration as the cell lines were not fully validate to be homozygously-tagged. nucleus generated from a SiR-DNA signal from a mask of the whole cell generated from a mEGFP-Nup signal. The mask for the nucleoplasm was created by eroding three pixels of the nuclear mask generated from a SiR-DNA signal. The Nup fluorescence intensity was quantified on these cytoplasmic and nucleoplasmic masks over time and then converted into the concentration. To calculate the total number of Nups in the cytoplasm and the nucleoplasm, the measured concentration was multiplied by the volume of the respective compartments. For the cytoplasm volume, we used the value that was measured previously using fluorescently-labelled 492 dextran 22 . The nucleoplasmic volume was quantified in each mEGFP-Nup knock-in cell line using a SiR-DNA signal as described previously ${ }^{12}$.

Core regions were predicted on the NE based on a previously described protocol using the core marker Lap-2 $\alpha^{12}$. Briefly, nuclear volume was segmented using SiR-DNA fluorescence 
volume was then divided into inner and outer volumes using the cutting plane that was constructed

498 from the largest eigenvector and the second one orthogonal to the first vector of the pixel

499 coordinates of the nuclear volume. Surface area of each nucleus was calculated and utilized to

500 adjust the size of the inner and outer core regions at individual time points. The previously defined

501 criteria for being core and non-core regions ${ }^{12}$ was applied. The position of inner and outer core

502 was determined with respect to the intersection point of the largest eigenvector on the nuclear

503 surface.

504

\section{Mathematical modeling for the nuclear pore assembly kinetics}

506 Previous EM data showed that, within 2 hours after anaphase onset, postmitotic assembly is the

507 dominant process in the non-core region, whereas the slower interphase assembly prevails in the 508 core region ${ }^{12}$. Assuming that this relation is also reflected in the live-cell Nup dynamics, we 509 derived a mathematical model. We assumed that the observed total fluorescence intensity in the 510 non-core, $n(t)$, and core region, $c(t)$, at time-point $t$ after anaphase onset is a linear combination

511 of the postmitotic and interphase assembly processes according to

$$
\begin{aligned}
& n(\mathrm{t})=\mathrm{f}_{\mathrm{N}} p m(\mathrm{t})+\left(1-\mathrm{f}_{\mathrm{N}}\right) \operatorname{ip}(\mathrm{t}) \\
& c(\mathrm{t})=\mathrm{f}_{\mathrm{C}} p m(\mathrm{t})+\left(1-\mathrm{f}_{\mathrm{C}}\right) \operatorname{ip}(\mathrm{t}),
\end{aligned}
$$

512 where $p m(t)$ and $i p(t)$ are the postmitotic and interphase assembly, respectively. The fraction of

513 postmitotic assembly in the non-core and core regions are denoted $f_{n}$ and $f_{c}$, respectively. To test

514 this assumption and obtain an estimate of the fractions, we used a phenomenological model that 515 accounts for the observed sigmoid-like kinetics

$$
\begin{gathered}
p m(t)=\frac{t^{n_{p}}}{t^{n_{p}}+K_{p}^{n_{p}}}, \quad \text { for } t \geq d \\
i p(t)=\frac{t^{n_{i}}}{t^{n_{i}}+K_{i}^{n_{i}}}, \quad \text { for } t \geq d
\end{gathered}
$$


and $p m(t<d)=i p(t<d)=0$. The parameters $n_{p}, K_{p}$ and $n_{i}, K_{i}$ characterize the postmitotic interphase and postmitotic assembly is $K_{i}$ and $K_{p}$, respectively. The parameter $d$ accounts for an additional delay in NPC core region assembly with respect to the non-core region due to the presence of kinetochore microtubule fibers ${ }^{25}$. For the non-core region $d=0$. For the core region $d$ was estimated from the data. to $\alpha_{2}=0.9$, is given by (see also Extended Data Fig. $4 \mathrm{a}$ )

$$
\begin{aligned}
& \Delta T_{p}=K_{p}\left(\left(\frac{\alpha_{2}}{1-\alpha_{2}}\right)^{\frac{1}{n_{p}}}-\left(\frac{\alpha_{1}}{1-\alpha_{1}}\right)^{\frac{1}{n_{p}}}\right) \\
& \Delta T_{i}=K_{i}\left(\left(\frac{\alpha_{2}}{1-\alpha_{2}}\right)^{\frac{1}{n_{i}}}-\left(\frac{\alpha_{1}}{1-\alpha_{1}}\right)^{\frac{1}{n_{i}}}\right) .
\end{aligned}
$$

In the model, we subtracted a background computed from the average of the first 3 time points.

526 All data is subsequently normalized to the mean value between 100 and 120 min after anaphase 527 onset. This yields corrected fluorescence intensities, $N\left(t_{j}\right)$ and $C\left(t_{j}\right)$. To find the model 528 parameters we minimized the mean squared distance between data and model for all the time points $M$

$$
\chi^{2}=\sum_{j=1}^{M}\left(\left(\frac{N\left(t_{j}\right)-n\left(t_{j}\right)}{\sigma_{N}\left(t_{j}\right)}\right)^{2}+\left(\frac{C\left(t_{j}\right)-c\left(t_{j}\right)}{\sigma_{C}\left(t_{j}\right)}\right)^{2}\right)
$$
where $\sigma_{N}\left(t_{j}\right)$ and $\sigma_{C}\left(t_{j}\right)$ are the respective standard deviation in the non-core and core region.

The parameter estimation has been performed in two steps. First, for each protein/cell-line,

532 we estimated the postmitotic fraction in the core and non-core region and the kinetic parameters.

533 All in all we computed 60 parameters ( 6 parameters per protein) from 4446 data points. We found 534 that the model well agrees with the data (Extended Data Figs. 2 and 3, $\mathrm{R}^{2}>0.99$ ). For the 
postmitotic fractions, we obtained on average $f_{n}=0.857[0.76,0.95]$ and $f_{c}=0.295[0.17,0.4]$, where the number in brackets indicates the $95 \%$ confidence interval as estimated using the profile

537 likelihood method ${ }^{36}$. Importantly, the obtained postmitotic fractions are well in agreement with 538 the previously reported estimates obtained from EM-data ${ }^{12}$. The delay in pore formation between 539 core and non-core region was estimated by systematically varying $d$ from 0 to 6 min in steps of 1 540 minute. A value of $d=2 \mathrm{~min}$, gave optimal result. In a second step we used the previously estimated average postmitotic fractions and $d$ and recomputed the kinetics parameters for each 542 protein. The model with reduced parameters gave very similar results. To verify if the choice of 543 common postmitotic fractions for all Nups is valid, we computed the Baysian information criterion 544 (BIC) according to the previous report ${ }^{37}$. The difference in BIC between the model with reduced 545 parameters, 42 parameters for all proteins, compared to the full model, 60 parameters, was -7 , 546 indicating that the model with reduced parameters is justified. The obtained parameter values are 547 listed in Extended Data Table 2.

Our validated model can be used to derive the underlying post-mitotic and interphase assembly kinetics. Using Eqs. 1-2 we obtain

$$
\begin{gathered}
i p(t)=\frac{f_{n} c(t)-f_{c} n(t)}{f_{n}-f_{c}} . \\
p m(t)=\frac{\left(1-f_{c}\right) n(t)-\left(1-f_{n}\right) c(t)}{f_{n}-f_{c}} .
\end{gathered}
$$

550 These equations can directly be applied to the experimental data $N(\mathrm{t})$ and $C(\mathrm{t})$ using the previously 551 estimated fraction $f_{n}$ and $f_{c}$.

In Fig. 3c we see a strong positive correlation for the average time point of assembly and the 553 duration of assembly. One can prove that such a relation is expected for an irreversible sequential 554 assembly mechanism with linear rate constants. Using the definition for average time and 555 duration $^{38}$, the average time-point of a protein binding at step $i$ is 


$$
\tau_{i}=\sum_{j=1}^{i} 1 / k_{j}
$$

where $k_{j}$ is the binding rate constant of a protein binding at step $j$ to the complex. The duration of assembly of a protein binding at step $i$ is given by

$$
\theta_{i}=2 \sqrt{\sum_{j=1}^{i} 1 / k_{j}^{2}} .
$$

From Eqs 10-11, it is clear that $\tau_{i+1}>\tau_{i}$ and $\theta_{i+1}>\theta_{i}$ for any parameter combinations. Therefore, late binding proteins show a longer duration of assembly.

\section{Structural modeling of NPC assembly pathway}

A model of the assembly pathway is defined by a series of static structures, including a static structure at each sampled time point along the assembly process. Therefore, we model the NPC assembly by first modeling static structures at each time point, independently from each other. We then enumerate alternative assembly pathways and rank them based on the static structure scores and plausibility of transitions between successive static structures.

\section{Integrative modeling of static structures at each time point}

The static structures are modeled by standard integrative structure modeling ${ }^{29}$, as follows.

\section{Representing a static structure model}

The time points correspond to times with available ET protein densities ${ }^{11}: 5 \mathrm{~min}, 6 \mathrm{~min}, 8 \mathrm{~min}, 10$ mins, and 15 min after anaphase onset. We divide the mature NPC structure (PDB 5a9q, 5ijo) into eight spokes and further divide each spoke into a set of rigid subcomplexes, including the Ycomplex, the inner ring Nup205-Nup155-Nup93 subcomplex, the inner ring Nup93-Nup188Nup155 subcomplex, and the central channel Nup62-Nup58-Nup54 subcomplex. For each domain, we coarse-grained the structure by grouping 10 consecutive amino acid residues into a single bead at the center of mass of those residues. Each subcomplex is represented as a rigid body. 
The NE is represented as a fixed toroid surface embedded in two parallel planes. Thus, the variables of the model include the Euclidean coordinates of the Nup subcomplexes and the copy number of each Nup subcomplex.

We set the inner pore diameter and minor radius of the pore at each time point to the mean of 581 previously determined NE cross sections ${ }^{11}$ with a pore diameter of $51.5 \mathrm{~nm}, 58.4 \mathrm{~nm}, 72.7 \mathrm{~nm}$, $58284.6 \mathrm{~nm}, 79.8 \mathrm{~nm}$, and $87 \mathrm{~nm}$; and minor radius of $21.4 \mathrm{~nm}, 21.2 \mathrm{~nm}, 21.5,20.3 \mathrm{~nm}, 17.1 \mathrm{~nm}$ and

$58315 \mathrm{~nm}$ for time points at $5 \mathrm{~min}, 6 \mathrm{~min}, 8 \mathrm{~min}, 10 \mathrm{~min}, 15 \mathrm{~min}$, and the mature pore, respectively.

585 The copy numbers of the NPC subcomplexes at each time point were restrained by a Gaussian 586 function with mean and variance determined by the single-cell traces presented in this study. The 587 relative likelihood of a set of copy numbers is proportional to the product of individual Gaussian 588 likelihoods.

Distances between pairs of Nups that are in contact with each other in the native NPC structure $^{26,27}$ were restrained by a harmonic Gō-like model ${ }^{39}$. Inter-subcomplex contacts within 5 $591 \mathrm{~nm}$ in the mature structure were restrained by a harmonic function (strength $0.01 \mathrm{kcal} / \mathrm{mol} \AA$ ). 592 Each Gō-like scoring term was scaled at each time point, from zero at the first time point to full 593 strength at the mature pore time point. Distances between all pairs of Nups were also restrained 594 by a harmonic excluded volume restraint (strength $0.01 \mathrm{kcal} / \mathrm{mol} \AA$ ). Proximity between Nup 595 domains containing a membrane interacting ALPS-motif and the NE was restrained by a harmonic 596 term (strength $0.1 \mathrm{kcal} / \mathrm{mol} \AA$ ), based on their sequences. Overlap between the Nups and NE 597 surface was avoided by imposing a harmonic repulsion between the Nups and NE surface (strength 598 of $0.01 \mathrm{kcal} / \mathrm{mol} \AA)$.

The shape of a static structure was restrained by a correlation coefficient between the model 600 and ET protein density ${ }^{11}$. The forward model density was represented by fitting each Nup 601 subcomplex with a Gaussian mixture model of two components per subcomplex copy using the 
gmconvert utility ${ }^{40}$. Similarly, the ET protein densities at each time point were represented with a

603 Gaussian mixture model with 150 components fit to the experimental density.

604

605

606

607

608

609

610

611

612

613

614

615

616

617

618

\section{Sampling static structure models}

A state of the NPC at any given time point is defined by the copy numbers and coordinates of its components. Only copy number assignments and structures consistent with the C-8 symmetries were sampled. In addition, we only sampled structures for the top 20-scoring Nup copy number combinations. Each sampling started with the mature pore structure, followed by applying $10^{6}$ Monte Carlo moves. These moves included rotational and translational perturbations to each Nup subcomplex, drawn from a uniform distribution in the range from -0.04 to +0.04 radians and from -4 to $+4 \AA$, respectively.

\section{Modeling the assembly pathway}

With the static structure models in hand, we connect them into complete alternative assembly pathways, as follows.

Each pathway is represented by a static structure at each sampled time point, starting with $\mathrm{t}=$ 5 min and culminating in the native structure; we do not model the completely disassembled NPC. The score of a pathway is the sum of the scores for the static structures on the pathway (defined above) and transitions between them. A transition score is uniform for all allowed transitions. A transition between two successive static structures is allowed if the subcomplexes in the first structure are included in the second structure. All possible pathways were enumerated, scored, and ranked. The best-scoring pathways were extracted for further analysis (Fig. 4).

\section{Sample size determination and statistical analysis}

For quantitative imaging in Fig. 1a, d, the data were from 4, 4, 4, 2, 3, 2, 3, and 2 independent experiments for Nup107, Seh1, Nup205, Nup93, Nup62, Nup214, Tpr and Nup358, respectively. STED imaging in Fig. 1b, c was from one independent experiment. For dynamic quantitative 
627 imaging in Fig. 2, the data were from 4, 4, 4, 2, 3, 4, 3, 2, 2, and 4 independent experiments for

628 Nup107, Seh1, Nup205, Nup93, Nup62, Nup214, Tpr, Nup358, Nup153, and Pom121,

629 respectively. Statistical analyses were performed only after all the data were taken. Sample sizes

630 for each experiment are indicated in figure legends. Sample sizes were based on pilot experiments

631 to determine the number of cells required to observe stable population averages with high

632 Pearson's correlation between replicates. Videos of dividing cells with rotating nuclei are removed

633 from the analysis, because we cannot properly assign the non-core and core regions.

634

635 Data and code availability

636 Fluorescence images will be available in the Image Data Resource (IDR;

637 https://idr.openmicroscopy.org/) or the Biolmage

638 (https://www.ebi.ac.uk/biostudies/BioImages/studies). Our integrative spatiotemporal model of

639 the postmitotic assembly of the human NPC is available in PDB-Dev (https://pdb-

640 dev.wwpdb.org/) under the accession code XX (PDB submission in progress). Integrative

641 Modeling Platform (IMP) is an open source program freely available under the LGPL license at 642 http://integrativemodeling.org; all input files, scripts, and output files are available at 643 http://integrativemodeling.org/npcassembly.

644

\section{Method references}

64632 Landry, J. J. et al. The genomic and transcriptomic landscape of a HeLa cell line. G3 $647 \quad$ (Bethesda) 3, 1213-1224 (2013).

64833 Farrants, H. et al. Chemogenetic Control of Nanobodies. Nat. Methods 17, 279-282 649 (2020).

65034 Tinevez, J. Y. et al. TrackMate: An open and extensible platform for single-particle 651 tracking. Methods 115, 80-90 (2017). 
65235 Pruggnaller, S., Mayr, M. \& Frangakis, A. S. A visualization and segmentation toolbox for

653 electron microscopy. J. Struct. Biol. 164, 161-165 (2008).

65436 Raue, A. et al. Structural and practical identifiability analysis of partially observed 655 dynamical models by exploiting the profile likelihood. Bioinformatics 25, 1923-1929 656 (2009).

65737 Kass, R.E. \& Raftery, A. E. Bayes Factors. J. Am. Stat. Assoc. 90, 773-795 (1995).

65838 Heinrich, R., Neel, B. G., \& Rapoport, T. A. Mathematical models of protein kinase signal 659 transduction. Mol. Cell 9, 957-70 (2002).

66039 Go, N. \& Abe, H. Noninteracting local-structure model of folding and unfolding transition 661 in globular proteins. I. Formulation. Biopolymers 20, 991-1011 (1981).

$66240 \quad$ Kawabata, T. Gaussian-input Gaussian mixture model for representing density maps and 663 atomic models. J. Struct. Biol. 203, 1-16 (2018).

664

665

Acknowledgments

666 We thank the EMBL Advanced Light Microscopy Facility (ALMF) for their support in STED 667 super-resolution microscopy; Ben Webb for help with IMP and Josh Baker-Lepain for managing 668 the Wynton computer cluster at QBI@UCSF. This work was supported by grants from the Baden 669 Wuerttemberg Foundation (J.E. and A.S.), NIH/NIGMS R01GM083960 (A.S.), NIH/NIGMS 670 P41GM109824 (A.S.), NIH/NIGMS R01GM112108 (A.S.) and the European Molecular Biology 671 Laboratory (EMBL; S.O., A.Z.P., A.R., M.J.H., M.K., A.C., B.K., J.E.). S.O. and A.R. were 672 further supported by the EMBL Interdisciplinary Postdoc Programme (EIPOD) under Marie Curie 673 Actions COFUND. S.O. was additionally supported by a JSPS fellowship (The Japan Society for 674 the Promotion of Science, postdoctoral fellowship for research abroad).

675 
677 SO, JOBT, AS, and JE designed the project. SO performed all the fluorescence microscopy

678 experiments and analyses. JOBT performed integrated structural modeling. AZP carried out

679 mathematical modeling for the nuclear pore assembly kinetics. AR developed an analysis pipeline

680 for nuclear pore density measurement. MJH established a computational image analysis pipeline

681 to quantify fluorescence intensities in non-core and core regions in 3D time-lapse images. M.K.,

682 A.C. and B.K. generated genome-edited cell lines. AS and JE supervised the work. SO, JOBT,

683 AS, and JE wrote the paper. All authors contributed to the analysis and interpretation of data and 684 provided input on the manuscript.

685

686 Competing interest declaration

687 The authors declare no competing interests.

688

689 Additional information

690 The manuscript contains supplementary material (Extended Data Figs. 1 to 4 and Extended Data 691 Tables 1 to 3 ). 